\title{
Particle movement and fluid behavior visualization using an optically transparent 3D-printed micro-hydrocyclone
}

Cite as: Biomicrofluidics 14, 064106 (2020); https://doi.org/10.1063/5.0025391

Submitted: 20 August 2020. Accepted: 04 November 2020 . Published Online: 19 November 2020

Maira Shakeel Syed, Fateme Mirakhorli, (D) Christopher Marquis, (D) Robert A. Taylor, and (iD) Majid Ebrahimi Warkiani
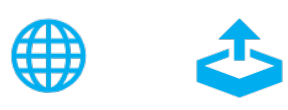

\section{ARTICLES YOU MAY BE INTERESTED IN}

Controllable microfluidic fabrication of microstructured functional materials

Biomicrofluidics 14, 061501 (2020); https://doi.org/10.1063/5.0027907

COVID-19 transmission in the first presidential debate in 2020

Physics of Fluids 32, 115125 (2020); https://doi.org/10.1063/5.0032847

\section{Biophysics Reviews}

\section{Publishing should be EASY, not stressful}

Find out how we're different! $>$ 


\title{
Particle movement and fluid behavior visualization using an optically transparent 3D-printed micro- hydrocyclone
}

\author{
Cite as: Biomicrofluidics 14, 064106 (2020); doi: 10.1063/5.0025391 \\ Submitted: 20 August 2020 . Accepted: 4 November 2020 . \\ Published Online: 19 November 2020
}

Maira Shakeel Syed, ${ }^{7}$ Fateme Mirakhorli, ${ }^{2}$ Christopher Marquis, ${ }^{3}$ (D) Robert A. Taylor, ${ }^{1,4}$ (D) and Majid Ebrahimi Warkiani ${ }^{2,5, a)}$ (1)

\begin{abstract}
AFFILIATIONS
${ }^{1}$ School of Mechanical and Manufacturing Engineering, University of New South Wales, Sydney, NSW 2052, Australia

${ }^{2}$ School of Biomedical Engineering, University of Technology Sydney, NSW 2007, Australia

${ }^{3}$ School of Biotechnology and Biomolecular Science, University of New South Wales, Sydney, NSW 2052, Australia

${ }^{4}$ School of Photovoltaic and Renewable Energy Engineering, University of New South Wales, NSW 2052, Australia

${ }^{5}$ Institute of Molecular Medicine, Sechenov First Moscow State Medical University, Moscow 119991, Russia
\end{abstract}

\begin{abstract}
${ }^{a)}$ Author to whom correspondence should be addressed: majid.warkiani@uts.edu.au
ABSTRACT

A hydrocyclone is a macroscale separation device employed in various industries, with many advantages, including high-throughput and low operational costs. Translating these advantages to microscale has been a challenge due to the microscale fabrication limitations that can be surmounted using 3D printing technology. Additionally, it is difficult to simulate the performance of real 3D-printed micro-hydrocyclones because of turbulent eddies and the deviations from the design due to printing resolution. To address these issues, we propose a new experimental method for the direct observation of particle motion in 3D printed micro-hydrocyclones. To do so, wax 3D printing and soft lithography were used in combination to construct a transparent micro-hydrocyclone in a single block of polydimethylsiloxane. A highspeed camera and fluorescent particles were employed to obtain clear in situ images and to confirm the presence of the vortex core. To showcase the use of this method, we demonstrate that a well-designed device can achieve a 95\% separation efficiency for a sample containing a mixture of (desired) stem cells and (undesired) microcarriers. Overall, we hope that the proposed method for the direct visualization of particle trajectories in micro-hydrocyclones will serve as a tool, which can be leveraged to accelerate the development of micro-hydrocyclones for biomedical applications.
\end{abstract}

\section{Published under license by AIP Publishing. https://doi.org/10.1063/5.0025391}

\section{INTRODUCTION}

Accurate size separation or fractionation of micro-particles is a critical step in several industrial processes, ${ }^{1}$ environmental assays, ${ }^{2}$ biotechnological sensing and production, ${ }^{3-5}$ and in numerous research activities. ${ }^{6,7}$ Numerous active and passive microfluidic systems have been developed to separate and/or concentrate microparticles and cells. Active separation techniques, including dielectrophoresis, acoustophoresis, and magnetophoresis, offer relatively fast and efficient particle sorting, but they are intricate and expensive. ${ }^{8}$ Passive techniques, including membrane-microfiltration and hydrodynamic sorting, are frequently utilized, but they are often slow and less efficient/selective methods. ${ }^{9,10}$ To address these issues, researchers have proposed new micro-separation approaches, including hydrodynamic filtration, ${ }^{11-13}$ deterministic lateral displacement (DLD), ${ }^{14}$ pinched flow fractionation, ${ }^{15,16}$ inertial microfluidics, ${ }^{17-20}$ shearinduced diffusion, ${ }^{21,22}$ and centrifugal microfluidics. ${ }^{23,24}$ Nevertheless, most of these devices suffer from clogging at high concentrations and have a relatively low throughput. ${ }^{25}$

One (previously) macro-scale separation technique that avoids these issues is the hydrocyclone. Macro-scale hydrocyclones have been widely used for decades to separate or classify big and heavy particles. ${ }^{26-28}$ In prior works, the authors (and others) have demonstrated the potential of shrinking hydrocyclones to the microscale to push toward the separation of micro-particles or cells. ${ }^{29-33}$ The authors believe that this will be transformative for 
biotechnological applications such as the cultivation of cells. In one particular application (investigated in the present work), to eliminate problems of current separation methods-such as cell damage (due to high shear stress) and high operating costs-there is a need for an alternative method to separate adherent cells from their suspension scaffolds, also known as microcarriers (MCs). ${ }^{34-36}$ Micro-hydrocyclones can be a proper substitute for these conventional separation methods, including downstream bulk process of tangential flow filtration (TFF), counter-flow centrifugation elutriation (CCE), or dead-end sieving. ${ }^{37,38}$ But, due to the fact that the hydrocyclone is inherently a complex $3 \mathrm{D}$ device, the miniaturization of hydrocyclones has been limited by the resolution of the manufacturing process.

With the advent of micro-fabrication and high-resolution additive manufacturing, however, it is now possible to "miniaturize" conventional systems and develop new technologies. High-resolution additive manufacturing, ${ }^{39,40}$ can-as of 2020-produce feature sizes down to $10 \mu \mathrm{m}$, ${ }^{39}$ which will enable the separation of microparticles/ cells. $^{41-43}$ In addition, the 3D-printing technology has recently crossed the threshold where such micro-scale parts can be accurately produced. $^{44-46}$ As it is now possible to do rapid prototyping of unique microdevices that can precisely manipulate particle flows on the microscale, ${ }^{47}$ our ability to directly observe and gain fundamental insights into such microparticle-laden flows has lagged.

In our previous study, we proposed a 3D-printed microhydrocyclone that could be applied to the primary harvesting of microalgae cells. ${ }^{31}$ In that study, however, the flow inside the microhydrocyclone could not be observed due to the opaque materials used in the device. However, we realized that without being able to visualize the particle movement and flow behavior inside a micro-hydrocyclone, we were quite dependent on our simulations (which required a lot of simplifying assumptions). The flow inside macro-scale hydrocyclones (Dc $>10 \mathrm{~mm}$ ) has been studied in detail using direct evidence from laser Doppler anemometry (LDA), particle imaging velocimetry (PIV), and other observation techniques. ${ }^{48-50}$ On the micro-scale, though, the flow field and particle behavior have never been directly observed. We believe that this is due to the complexity of the fabrication process and the restrictions of empirical observations.

To address this, we report a two-step workflow for the fabrication of transparent micro-hydrocyclones using the $3 \mathrm{D}$ wax printing technique. A $3 \mathrm{D}$ printed sacrificial mold is utilized to fabricate the micro-hydrocyclone with high resolution $(6 \mu \mathrm{m}-25 \mu \mathrm{m}$ in the $\mathrm{Z}$ direction) using soft lithography. Polydimethylsiloxane (PDMS), which is commonly used for $2 \mathrm{D}$ microscale devices, was selected to produce the final device because of its optical transparency. The transparent micro-hydrocyclone is used for flow visualization and for particle tracking. In addition, we have used this device to showcase the separation of stem cells from microcarriers while observing cell and microcarrier movement. Due to the constant improvement in the $3 \mathrm{D}$ printers' resolution, it is hoped that designs similar to the one proposed in the present study can be extended to various applications required for low-cost and high-throughput operations.

\section{DESIGN AND THEORETICAL ANALYSIS}

The tangential injection of a fluid into a confined space produces a swirling flow field in a cyclone, leading to flow separation.
This is possible because of the presence of double vortex flow field in which some of the flow goes out the top of the cyclone (e.g., the overflow adjacent to the inlets), and some goes out the bottom (e.g., the underflow) of the cyclone. The inner upward vortex (i.e., the secondary vortex) counterbalances the outer downward vortex (the primary vortex). This swirling flow gives rise to the centrifugal force that pushes the larger/denser particles to the wall, while the smaller/lighter particles are dragged to the low-pressure zone in the center, where they can be carried by the secondary vortex to the overflow exit. Accordingly, a particle having a diameter $\left(D_{p}\right)$ and density $\left(\rho_{p}\right)$ will have three forces acting on it: (i) the centrifugal force $\left(F_{c}\right)$, an outward radial direction due to the tangential velocity $\left(v_{t}\right)$; (ii) the buoyant force $\left(F_{b}\right)$, an inward radial direction that is due to the density difference of the fluid $\left(\rho_{f}\right)$ and the particle $\left(\rho_{p}\right)$; and (iii) the drag force $\left(F_{d}\right)$, which causes the particles to follow the flow inward or outward, depending upon the local radial velocity $\left(v_{r}\right)$ of the particle so that it always opposes the particle movement due to the fluid viscosity $\mu$. The magnitude of the drag force depends on the fluid viscosity, $\mu$, the particle size and shape, and the turbulence flow intensity. These forces are mathematically defined by Eqs. (1)-(3). ${ }^{51}$ Other forces such as pressure gradient force, Saffman lift force $(\sim 15$ orders of magnitude smaller than the drag force), and Brownian motion can be ignored due to extremely small magnitudes compared to other forces, ${ }^{52}$

$$
\begin{gathered}
F_{c}=m \frac{v_{t}^{2}}{r}=\frac{\pi \rho_{p} D_{p}^{3} \frac{v_{t}^{2}}{r}}{6}, \\
F_{b}=-V_{p} \rho_{f} \frac{v_{t}^{2}}{r}=-\frac{\pi D_{p}^{3}}{6} \frac{v_{t}^{2}}{r} \rho_{f}, \\
F_{d}=-3 \pi D_{p} \mu v_{r} .
\end{gathered}
$$

The separation performance and flow physics inside a hydrocyclone are broadly dependent on its design parameters. ${ }^{53,54}$ The geometric dimensions are provided in Table I and illustrated in the inset of Fig. 1 , where $D_{c}, L_{1}, L_{2}, D_{i}, D_{o}, D_{u}$, and $h$, are cylindricalsection diameter, cylindrical-section length, conical-section length, feed inlet diameter, overflow diameter, and underflow diameter, respectively. Although there are a lot of parameters, only a few key design ratios, dependent on the cylindrical section diameter, $D_{c}$, are needed to specify the geometry of a hydrocyclone. For example, the Bradley ratio and Reitema's ratio provide simplified design rules for fabricating hydrocyclones. ${ }^{55}$ In this study, our micro-hydrocyclone was designed based on Bradley's geometric ratio ${ }^{56}$ as the Bradley model achieves a smaller cut size than Reitema, thereby more suitable for our purpose. ${ }^{56}$

TABLE I. Geometric parameters of the fabricated micro-hydrocyclone.

\begin{tabular}{lccccccc}
\hline \hline $\begin{array}{l}\text { Parameters } \\
(\mathrm{mm})\end{array}$ & $D_{c}$ & $L_{1}$ & $L_{2}$ & $D_{i}$ & $D_{o}$ & $D_{u}$ & $\begin{array}{c}\text { No. of } \\
\text { inlets }\end{array}$ \\
\hline Our design & 3 & 2.16 & 23.16 & 0.428 & 0.40 & 0.65 & 2 \\
\hline \hline
\end{tabular}




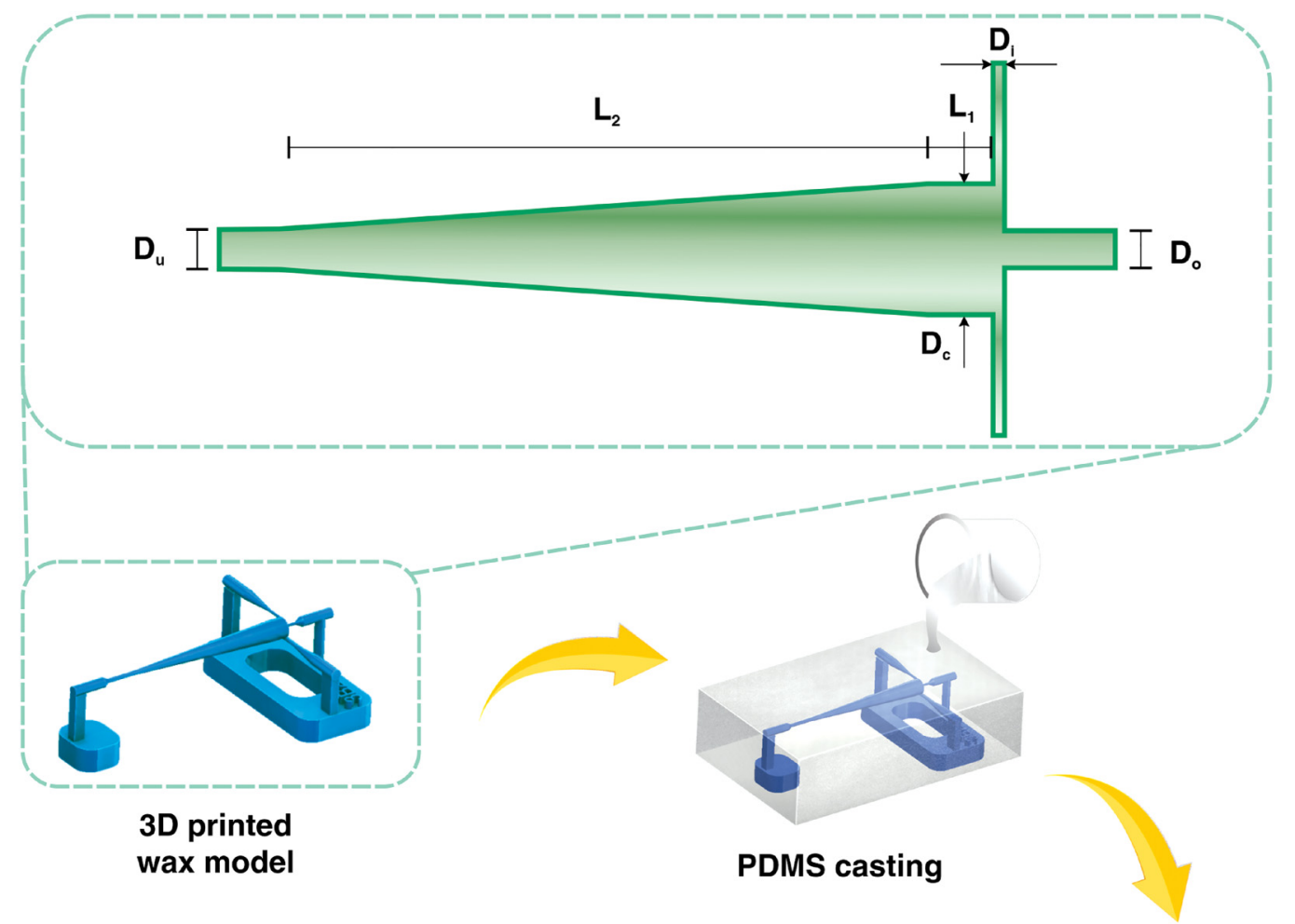

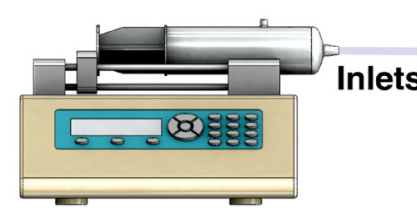

Syringe pump

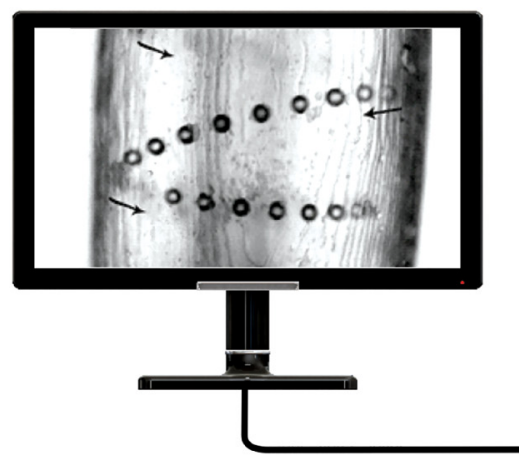

Particle visualization

\section{Light source}

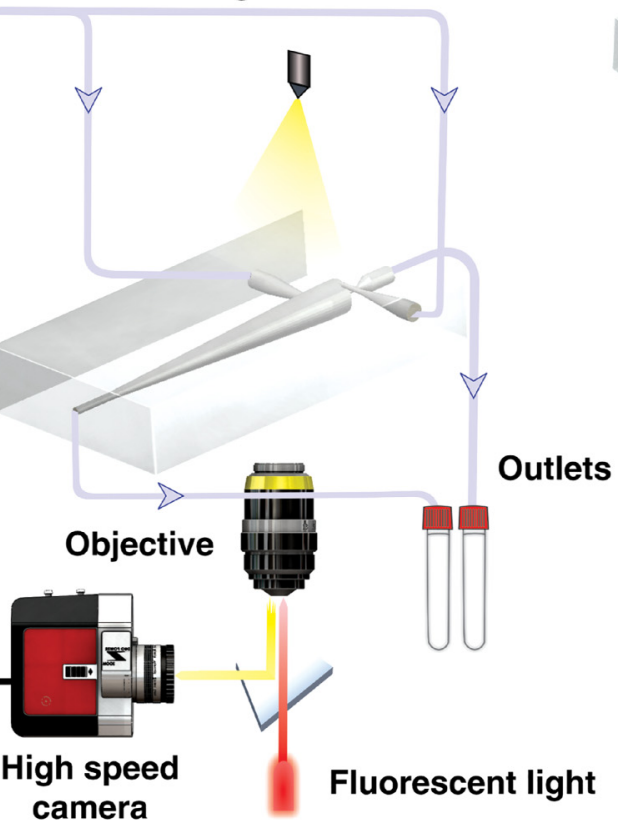

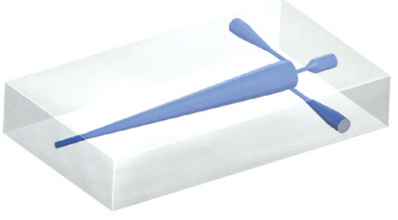

Cured PDMS with wax model

FIG. 1. Schematic illustration of the workflow for the fabrication and characterization of a transparent PDMS-made micro-hydrocyclone. The cyclone body was first printed using a wax 3D printer (critical dimensions are illustrated in the inset) and then positioned into a casting box for soft lithography. Afterward, wax materials were removed from the PDMS block, and the transparent micro-hydrocyclone was fabricated by cutting extra PDMS parts using a blade. The micro-hydrocyclone was then placed horizontally on the microscope for fluorescent, bright field, and high-speed camera imaging and microscopy. 


\section{MATERIALS AND METHODS}

\section{A. Numerical simulation}

Despite the simplicity of the micro-hydrocyclone geometry, the high-velocity turbulent swirl flow inside the microhydrocyclone makes the CFD simulation of the flow difficult. As the fluid flow inside the micro-hydrocyclone is a highly anisotropic turbulent flow, turbulence models are needed to carry out the numerical simulations. Based on prior studies, ${ }^{57,58}$ the Reynolds stress model (RSM) was chosen as the most appropriate approach for simulating the flow in a micro-hydrocyclone. To this end, the CAD model of the micro-hydrocyclone was drafted using SolidWorks 2016 (Dassault Systèmes, USA). Afterward, this model was discretized by ICEM 19.2 (ANSYS). Using FLUENT 19.2 (ANSYS), mesh independence analysis was performed to obtain the optimum size of grids. In this model, the particle-liquid behavior was simulated under steady flow conditions. Eulerian and Lagrangian methods were employed to simulate the continuous phase and particle trajectories, respectively. A uniform inlet velocity $(\mathrm{V}=5 \mathrm{~m} / \mathrm{s})$ and atmospheric pressure boundary conditions were applied for both inlets and outlets, respectively. Due to the high-pressure gradients of the double-vortex flow inside the micro-hydrocyclone, an algorithm is required to compute the pressure profile. For this purpose, the PRESTO (pressure staggered option) scheme was used. Furthermore, the SIMPLE (semi-implicit method pressure-linked equations) algorithm was employed for the pressure velocity coupling and momentum equations. Based on a prior study, the turbulent kinetic energy needs to be second-order, and the Reynolds stresses need to be the first order. ${ }^{59}$ To ensure the accuracy of the solution, the criterion for the convergence of the scaled residuals was set to $1 \times 10^{-5}$. The particle trajectories were calculated using the discrete phase model (DPM), considering the volume fraction of the particles to be less than $10 \%$. In the computation of the particle trajectories, the program keeps track of momentum exchange between the particle and the surrounding continuous phase. For the two-way coupled multi-phase flow, the calculation of the continuous and discrete phase flows was alternated, and a converged solution was achieved.

Particles reaching the overflow were set to "escape," while those reaching the underflow were set to "trapped". The hydrodynamic profiles of the particles, velocity, and pressure contours on different planes were observed. Finally, the separation efficiency was calculated by Eq. (4),

$$
\text { Separation efficiency }(\%)=\frac{\text { No. of particles trapped }}{\text { Total No. of particles }(\text { trapped }+ \text { escaped })} \times 100 \text {. }
$$

\section{B. Device fabrication}

The micro-hydrocyclone model was printed with a SolidscapeVR MAX2 printer (Solidscape, Inc., USA). Due to long and thin overhanging structures, some additional supports were added to the design without affecting the main micro-hydrocyclone structure. ${ }^{44}$ The printer mentioned above uses a combination of additive and subtractive manufacturing to make an object by developing successive 2D layers. Each layer is created using a direct writing technique, wherein molten wax droplets deposit on the layer and solidify by cooling on impact. The printing resolution after comparison of surface finish was found to be (a) at the highest resolution up to $6 \mu \mathrm{m}$ and (b) at the coarser setting, $25 \mu \mathrm{m}$. When the coarser resolution setting was used, the test models were printed at $25 \mu \mathrm{m}$ resolution taking approximately $10 \mathrm{~h}$. The finer settings took $\sim$ five times longer (e.g., $\sim 50 \mathrm{~h}$ of the printing time).

The final main structure and the surrounding structure were printed in the $3 \mathrm{Z}$ Model material and $3 \mathrm{Z}$ Support material (from Solidscape Inc.), respectively. After the printing completion, the part was removed from the build plate by putting it on a hot plate at $60{ }^{\circ} \mathrm{C}$. Thereupon, the detached part was immersed in a support removal solvent (Vantage Specialty Chemicals, USA) at $60^{\circ} \mathrm{C}$ for $30 \mathrm{~min}$ to remove the support material. Afterward, the product was placed on a specially designed PDMS casting box with polydimethylsiloxane (PDMS, Sylgard 184 Silicone Elastomer Kit, Dow Corning) poured on top of it. The PDMS was mixed at a ratio of
7:1 (base: curing agent). It is important to note that a lower mixing ratio was used than the usual 10:1 to make the PDMS harder (i.e., a higher bulk modulus) so that it will be less deformable under the high flow rates. Then, the assembly was degassed in a vacuum chamber, followed by a vacuum curing at $65-75^{\circ} \mathrm{C}$ for $3 \mathrm{~h}$. The cured PDMS containing the wax model was then sliced at the beginning point of the inlets and outlets, perpendicularly. Finally, the $3 \mathrm{D}$ printed wax model was molten/drained away from the inside the PDMS by placing the device in an oven (at $120^{\circ} \mathrm{C}$ for $2 \mathrm{~h}$, noting that the melting temperature of the wax is $95-120^{\circ} \mathrm{C}$ ). To remove the residual wax, the device was flushed with acetone (Fig. S1 in the supplementary material). A schematic illustration of the fabrication process is depicted in Fig. 1.

\section{Sample preparation}

To investigate the device performance, the suspension of fluorescent microparticles (Fluoresbrite Microspheres, Polysciences Inc, Singapore) with three different sizes of 3,10 , and $100 \mu \mathrm{m}$ and mammalian cells was utilized. The particle-liquid suspensions were prepared by mixing the microparticles in a buffer solution consisting of $1 \times$ phosphate buffered saline (PBS), 2 mM EDTA supplemented with $0.5 \%$ bovine serum albumin (BSA). The mesenchymal stem cells (MSCs) used in the present study were cultured in a T75 tissue culture flask and later inside a $250 \mathrm{ml}$ spinner flask (Corning) with its specific culture media which was MEM-Alpha (Gibco, USA) supplemented with $10 \% \mathrm{v} / \mathrm{v}$ fetal bovine serum (FBS, Gibco, USA) and 
1\% v/v Pen-Strep (Pen-Strep Solution, Biological Industries, Israel). To perform a cell count, $5 \mu$ l of Solution- 13 stain containing acridine orange and 4',6-diamidino-2-phenylindole (DAPI) (Solution 13 AO-DAPI $1 \mathrm{ml}$, Chemometec, Denmark) was spiked into $100 \mu \mathrm{l}$ of the suspended cell sample. The viable cell count (three measurements) was measured with a Nucleocounter (Nucleocounter NC-250, Chemometec, Denmark).

For the suspension cell culture, we used $250 \mathrm{ml}$ spinner flasks and polystyrene microcarriers (MCs) (Sigma with an average size of $180 \mu \mathrm{m}$ ) throughout the experiments. The concentration of the microcarrier stock was $10 \mathrm{mg} / \mathrm{ml}$ of beads in PBS and the concentration of the MCs was $2 \mathrm{mg} / \mathrm{ml}$. The polystyrene MCs were seeded with $2.4 \times 10^{4}$ cells $/ \mathrm{ml}$ of the culture media. The spinner flask was agitated at $38 \mathrm{rpm}$. Cells were cultured on MCs for seven days in an incubator at $37^{\circ} \mathrm{C}$ and $5 \% \mathrm{CO}_{2}$ level, in which $50 \%$ fresh culture medium was exchanged for all spinner cultures on days 2,4 , and 6 .

\section{Cell counting and analysis}

Before and after running the media through the microhydrocyclone, the cell sizes, concentrations, and viability were measured by a flow cytometer using propidium iodide dye. For verification, the traditional Trypan blue exclusion test was also performed using a hemocytometer, and it was found that the results agreed with results obtained using the flow cytometry.

\section{E. Experimental setup and procedures}

Before testing with cells, the micro-hydrocyclone was characterized by surrogate polystyrene fluorescent microparticles at different flow rates. BSA was added to avoid microparticle adhesion to micro-hydrocyclone walls and tubing. To dissociate any aggregates, the suspension was sonicated for an hour in a cold water bath and stirred regularly during the experiments using a vortex mixer to ensure homogeneity.

For the in situ characterization of the flow, the device was positioned horizontally on an inverted epifluorescence microscope (Olympus IX73 inverted microscope with DP80 camera) using a high-speed camera (FASTCAM SA5, Photron Inc., USA). The microparticle suspensions were injected into the microhydrocyclone using a peristaltic pump (Shenchen, LabV1, China), and the results were analyzed using Fiji software (https://fiji.sc/). For easy identification of the particle sizes, different colored particles were chosen (i.e., 3 and $10 \mu \mathrm{m}$ appeared green in blue light, whereas the $100 \mu \mathrm{m}$ particles looked red in green light). Particle traces and movements through the micro-hydrocyclone were monitored using a high-speed and fluorescent camera at different flow rates. To obtain this, certain frames using the high-speed camera can be viewed in series to track the particle as it swirls through the micro-hydrocyclone body and flows down to the underflow. Due to the difference in the length scales of the particles and the overall dimensions of the micro-hydrocyclone, the whole length could not be viewed. Of course, at lower magnifications, the particles cannot be imaged individually. As a trade-off, a low magnification lens (i.e., $2 \times$ ) was used with the Olympus microscope and traces of larger diameter particles (i.e., $\sim 180 \mu \mathrm{m}$ particles) were analyzed. For smaller particles, a much higher magnification was required, for which the micro-hydrocyclone width could not be entirely seen.
The micro-hydrocyclone was mounted horizontally on the microscope focused near the wall close to the microscope lens and the videos were recorded by the high-speed camera at 8882 frames per second (fps). In order to depict particle trajectories, the difference between each frame of the video file and a reference frame was evaluated using Fiji software. Since the camera was focused near the micro-hydrocyclone wall, some parts of the particle trace are missing as the particle swirled away from the level of focusing. The trajectory resumed as the particle completed its rotation and came back into focus (Video S1 in the supplementary material).

After the characterization of the device with the microparticles, the device was evaluated using MSCs and MCs. All the cellbased studies were performed inside a biosafety cabinet to avoid any contamination. The devices and tubing were first autoclaved and flushed with $70 \%$ ethanol for $1 \mathrm{~h}$ and washed out with sterile distilled water. The cell culture was then fed into the microhydrocyclone through the peristaltic pump. Samples collected from initial culture flask and micro-hydrocyclone outlets were used for MSC/MC counts and viability analysis.

For quantitative analysis, the discharge from both outlets was collected in different Falcon tubes and microbeads concentration before and after separation was determined with a hemocytometer. Based on these particle counts, the efficiency of microhydrocyclone is determined by Eq. (5),

$$
\text { Hydrocyclone efficiency }=1-\frac{X_{o}}{X} \text {, }
$$

where $X_{o}$ and $X$ are the concentrations of overflow stream and the feed, respectively.

\section{RESULTS AND DISCUSSION}

\section{A. Simulation results}

Figure 2 illustrates the contours of pressure magnitude, velocity, and particle distribution along the horizontal (near the top of the cylindrical section) and vertical planes (along the central axis) of a micro-hydrocyclone. Since the outlets were assumed to be open to the atmosphere, and that an inner upward vortex flow formed (all these results were confirmed for the first time by experimental evidence, as illustrated in Sec. IV B), there is a great pressure gradient where the fluid enters the micro-hydrocyclone, i.e., regions close to the inlets, and by moving toward outlets, the pressure near the micro-hydrocyclsone wall decreases. Consequently, an air core region with low pressures is developed within the micro-hydrocyclone body, as shown in Fig. 2(a). As the flow velocity inside the micro-hydrocyclone is directly related to the centrifugal forces acting on the particles caused by the swirling flow, the analysis of the flow velocity contours is of great importance. On the top plane of the micro-hydrocyclone, there was a drastic increase in the velocity magnitude as the fluid passed through the inlet pipes. However, as the fluid flows into the micro-hydrocyclone body and swirls around the micro-hydrocyclone walls, the velocity decreased gradually [Fig. 2(b)]. The high velocities in the cylindrical section of the micro-hydrocyclone increase $F_{c}$ acting on the particles and help to push the bigger particles toward the walls. A very low velocity region (in dark blue and yellow) [Fig. 2(c)] in the 
A)

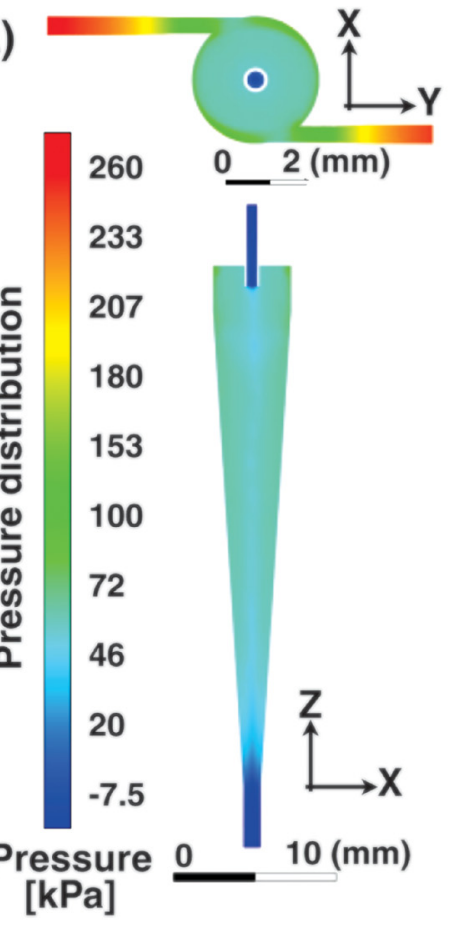

B)

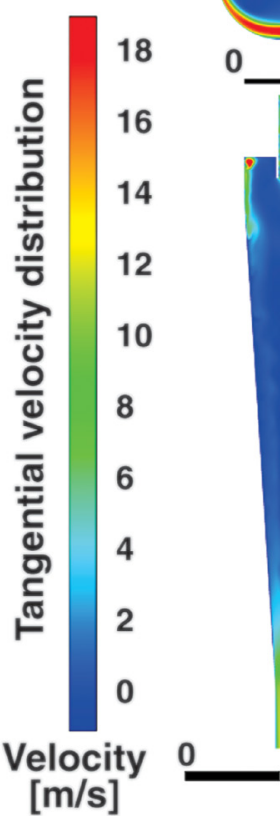

C)

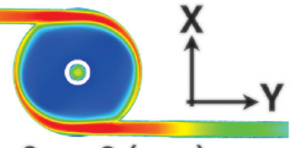

c)

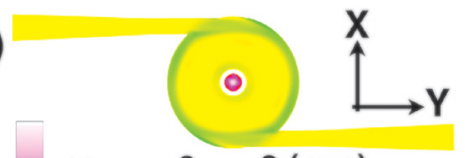

12

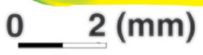

올

4

0

음
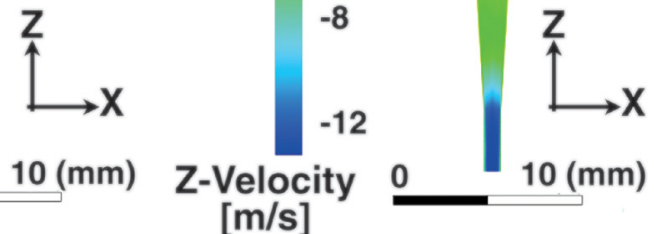

$-12$

$10(\mathrm{~mm})$

D)

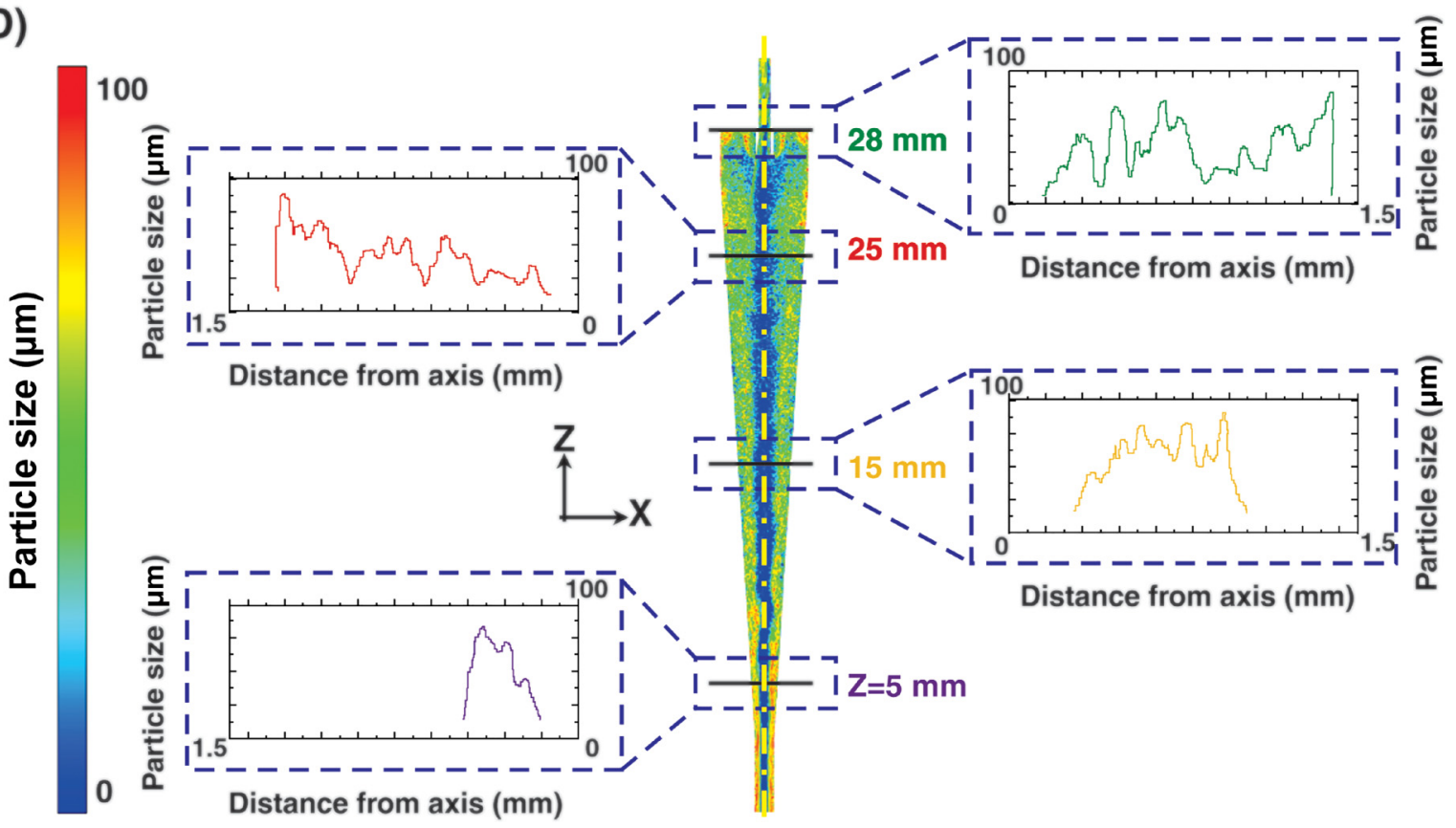

FIG. 2. (a) Pressure contours in the computational model of micro-hydrocyclone on the top and front planes. (b) Velocity magnitude contour on the top and front plane at the feed velocity $V=5 \mathrm{~m} / \mathrm{s}(Q=\sim 43 \mathrm{ml} / \mathrm{min}$ for each inlet). (c) Contours of Y-velocity (axial velocity) on the top and front planes. Positive velocities indicate upward fluid flow, whereas the negative velocities show the downward flow. (d) The distribution of particles with varying sizes on the front plane and the spreading of particles on different planes along with the height of the micro-hydrocyclone shown in the insets ( 0 is showing the position on the yellow line passing through the micro hydrocyclone center). 
middle of the cyclone can also be observed, depicting the air core region (i.e., the center of the swirling flow). This also depicts the presence of two vortices, the negative Y-velocity magnitudes show the downward flow towards the underflow (outer vortex), while the positive magnitudes represent the flow toward the overflow (inner vortex).

Analysis of particle trajectory within the micro-hydrocyclone chamber can give insight toward understanding the concept of the separation efficiency of the micro-hydrocyclone. In this regard, the distribution of particles with a size variety on the vertical plane is demonstrated in Fig. 2(d). The planes indicated in Fig. 2(d) are further elaborated for particle size distribution in the graph presented in the insets. For $y=28 \mathrm{~mm}$, which is near the microhydrocyclone inlet, the particles are spread out randomly through the width of the micro-hydrocyclone. At $y=25 \mathrm{~mm}$, the peak of the larger particles shifts towards the micro-hydrocyclone walls, while the smaller particles are still present near the microhydrocyclone center. A similar trend holds at the plane at $y=15 \mathrm{~mm}$, except that the number of smaller particles is lower than the previous plane, indicating that many of the smaller particles are caught up by the inner vortex flow approaching the overflow. This is also evident in Fig. 2(c) as the contour illustrates some positive magnitudes of $y$-velocity indicating the upward flow, between the planes $y=25 \mathrm{~mm}$ and $15 \mathrm{~mm}$. Please note that the $y$ axis of Fig. 2(d) indicates particle size, with a value of "0" denoting that no particles were observed in that location.

\section{B. Experimental results}

Previous simulation studies ${ }^{60,61}$ revealed that separation efficiency is directly affected by the inner vortex flow. Since the flow enters from two opposite sides in the micro-hydrocyclone, a

\section{Flow rate $=10 \mathrm{ml} / \mathrm{min}$}
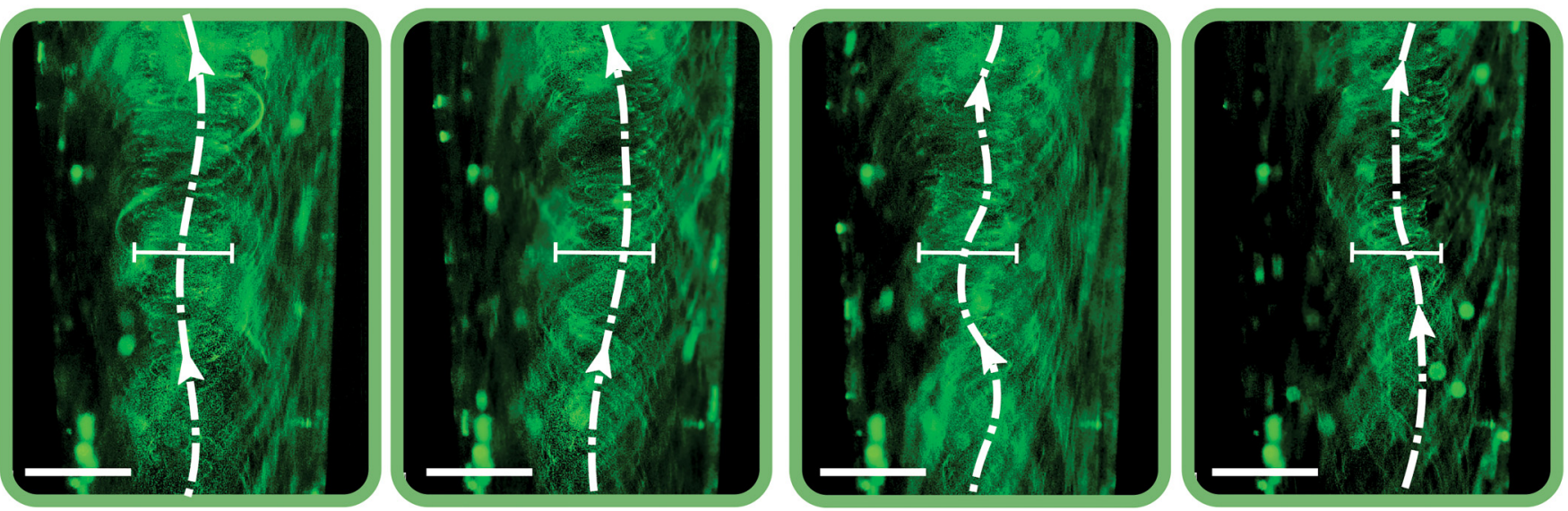

\section{Flow rate $=70 \mathrm{ml} / \mathrm{min}$}
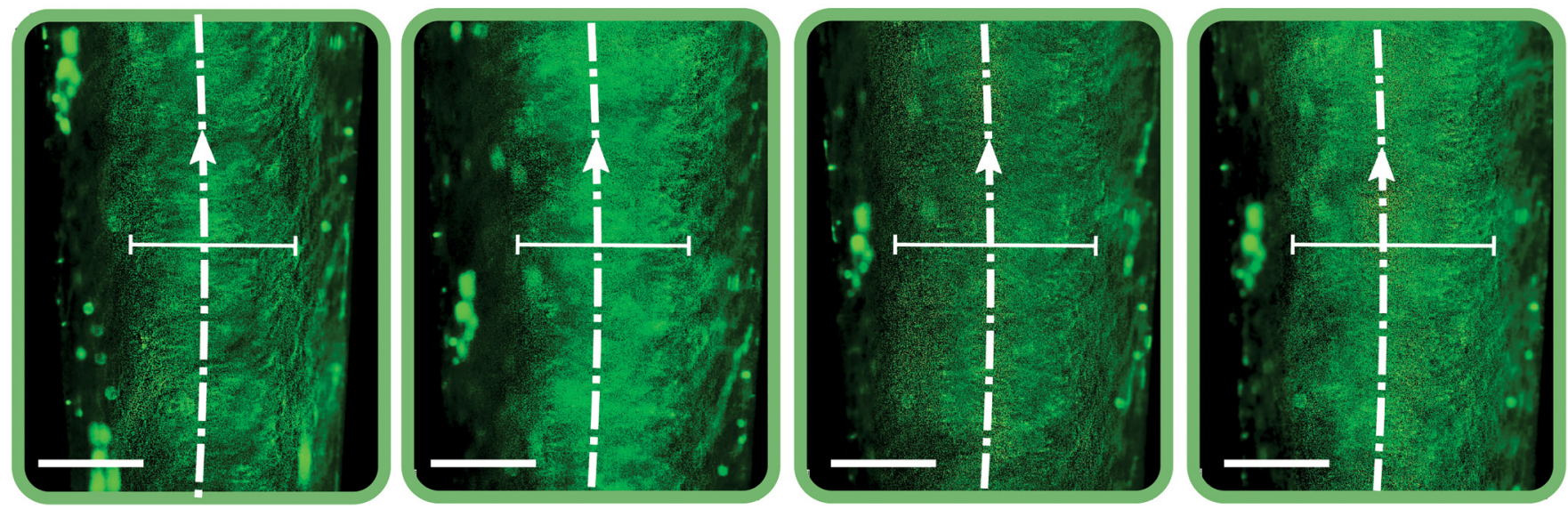

FIG. 3. Microscopic picture of the inner upward vortex inside a micro-hydrocyclone at two different flow rates of 10 and $70 \mathrm{ml} / \mathrm{min}$, which is obtained using $3 \mu \mathrm{m}$ fluorescent particles. At the lower flow rate of $10 \mathrm{ml} / \mathrm{min}$, the inner upward (core) vortex fluctuates along the cyclone axis, whereas at the higher flow rate of $70 \mathrm{~m} / \mathrm{min}$, it becomes more stable. Scale bar, $1 \mathrm{~mm}$. 
symmetrical flow field develops through the entire chamber of micro-hydrocyclone, leading to a "stable" inner vortex flow. Subsequently, two distinguished flows that carry different particle sizes can be effectively segregated into the lighter flow (e.g., an inner upward vortex flow containing finer, lighter particles) and the outer vortex flow (e.g., a downward flow containing bigger, denser particles). Our observations (see Fig. 3), however, revealed that at lower flow rates, the inner upward vortex flow tends to meander along the vertical axis of the double inlet microhydrocyclone. By increasing the flow rate from 10 to $70 \mathrm{ml} / \mathrm{min}$, the inner vortex flow becomes more stable, and its diameter gradually increases, as shown in Fig. 3 (Video S2 in the supplementary material). This helps explain why an increase in the inlet flow rate elevates the separation efficiency [Fig. 6(b)]. ${ }^{62}$

As was mentioned in numerical results, particles swirl within the micro-hydrocyclone during their path to the outlet (Fig. S2A in the supplementary material). Using the process of capturing the particle trajectory and motion by the high speed camera, as explained in Sec. III E, the rotation and velocity of the particles potentially can be calculated for comparison with the numerical simulation. As illustrated in Fig. 4, for two flow rates of 30 and $70 \mathrm{ml} / \mathrm{min}$, due to the turbulence, the path followed, and the number of turns, each particle follows a slightly different path through the same section. The previous studies ${ }^{63,64}$ revealed that, by increasing the size of a particle, the time required for a particle to leave the chamber increases. Also the density is proved to be another influential factor in the residence time. In this study, the effect of the flow rate on particle movements is investigated. According to the traces, in lower flow rates, particles are more likely to move back and forth through the micro-hydrocyclone chamber, which results in the increase in the residence time. Therefore, the higher the flow rate is, the lower the residence time becomes. Shorter residence time can potentially moderate the shear stress levels applied to the cells inside the micro-hydrocyclone. ${ }^{65}$

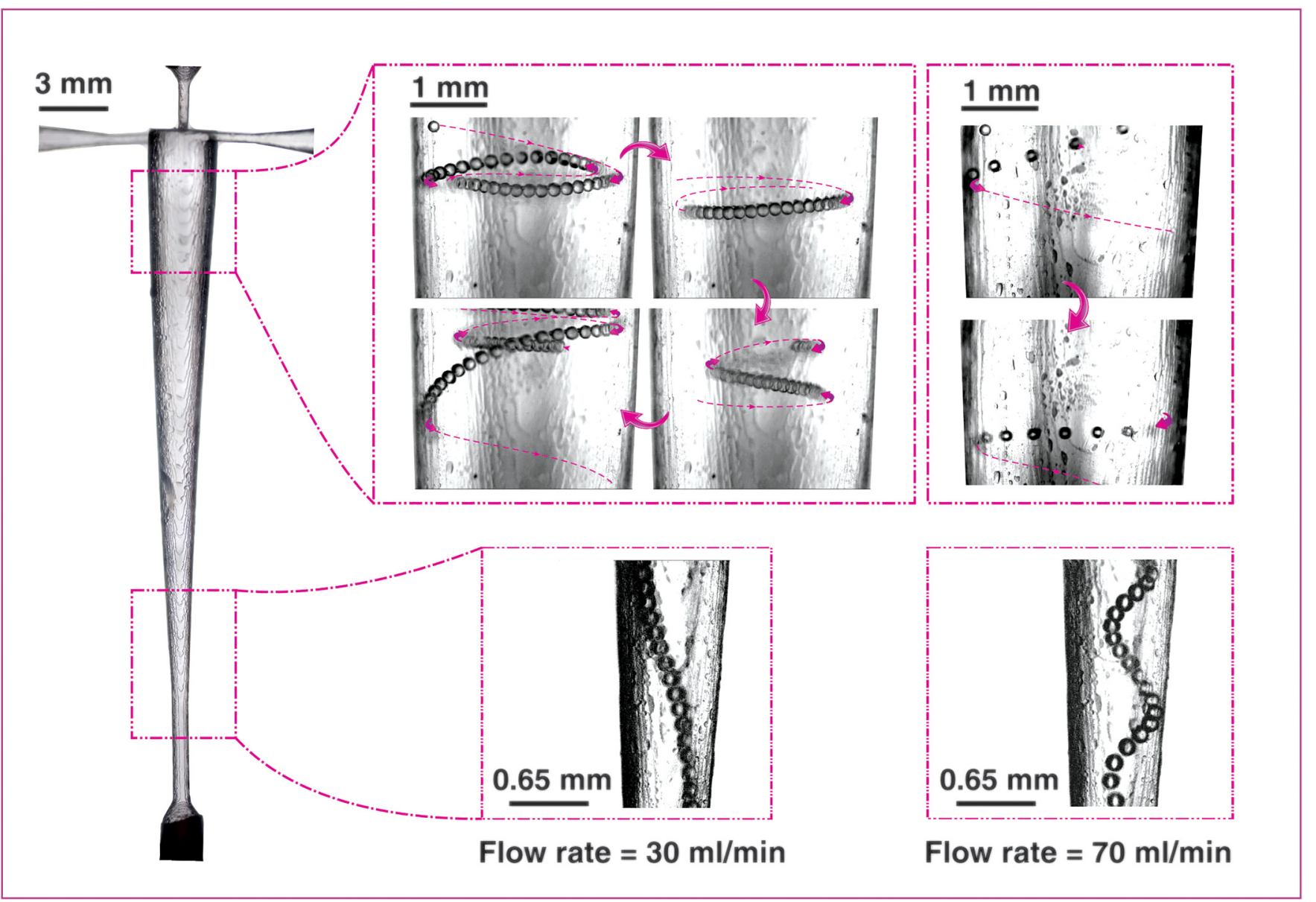

FIG. 4. Movement of $180 \mu \mathrm{m}$ particle at two different flow rates of 30 and $70 \mathrm{ml} / \mathrm{min}$. The pink dashed lines show only the unfocused particle trajectories within the microhydrocyclone. Based on the trajectories, in lower flow rates, particles are more likely to move back and forth through the micro-hydrocyclone chamber and fluctuate around the vertical axis, which results in the increase in the residence time. 
Small scale cyclones are proven to be useful devices for highthroughput cell separation and have been previously utilized for cell/particle separation. ${ }^{31}$ To do this, we initially characterized our micro-hydrocyclones using surrogate fluorescent particles (10 and $100 \mu \mathrm{m})$ to find the optimum separation efficiencies. These particle sizes were selected to mimic the separation of MCs from MSCs-an application which requires a good solution in the biotechnology industry. ${ }^{66}$ Similar to the previous section, the micro-hydrocyclone was mounted horizontally [Fig. 5(a)] on the microscope stage to obtain the particle traces using a sensitive CCD camera. The particle trajectories imaged at the feed flow rate of $75 \mathrm{ml} / \mathrm{min}$ at vertical location $\mathrm{y}=2 \mathrm{~mm}$ and $30 \mathrm{~mm}$ for underflow and overflow, respectively. At this flow rate, many of $10 \mu \mathrm{m}$ particles exited through both outlets due to insufficient forces acting on the particles [Fig. 5(b)]. On the contrary, almost all of the $100 \mu \mathrm{m}$ particles exited through the underflow, and no particle traces could be seen at the overflow [Fig. 5(c)]. Comparing these results with the simulation results [Fig. 2(d)], the same trend of separation for both particle sizes is observed, at a similar flow rate/feed velocity. Thus, the experimental results validate the simulation. These results show the potential application of the micro-hydrocyclone when the fractionation of different sizes of particles/cells is required (Fig. S2C and Video S3 in the supplementary material).

A)

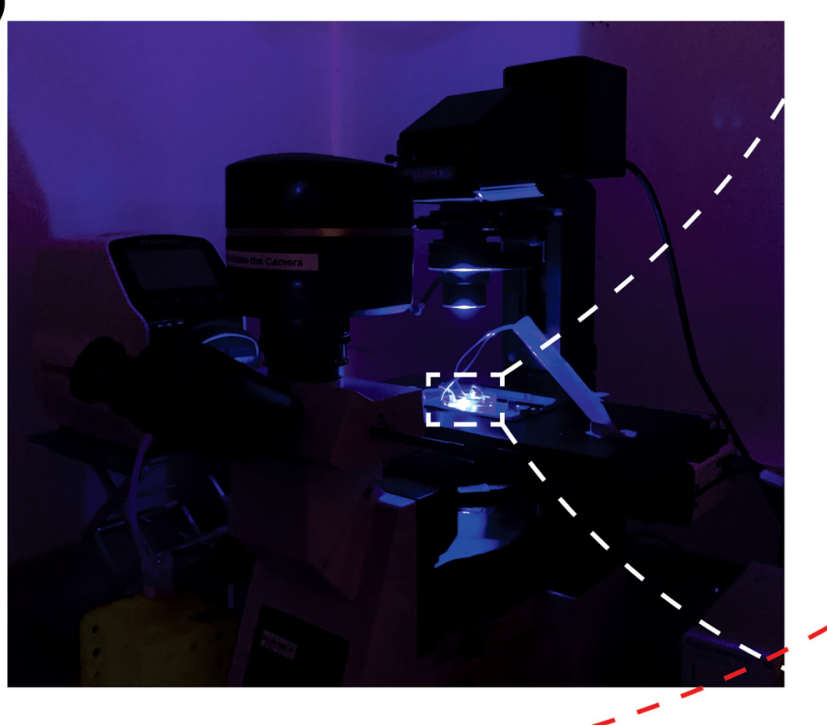

B)

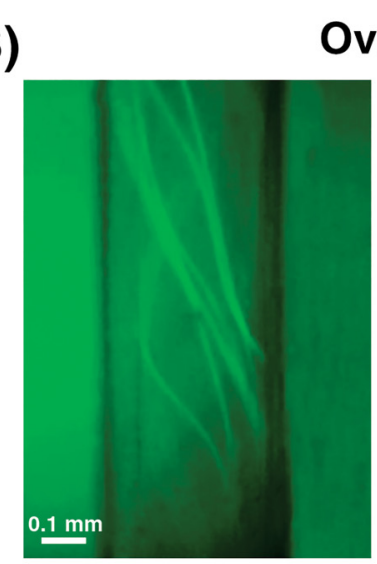

$10 \mu \mathrm{m}$
Overflow $-\bar{z}=30 \mathrm{~mm}$

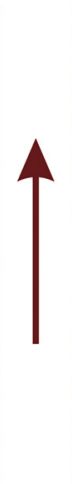

$0.1 \mathrm{~mm}$

$100 \mu \mathrm{m}$

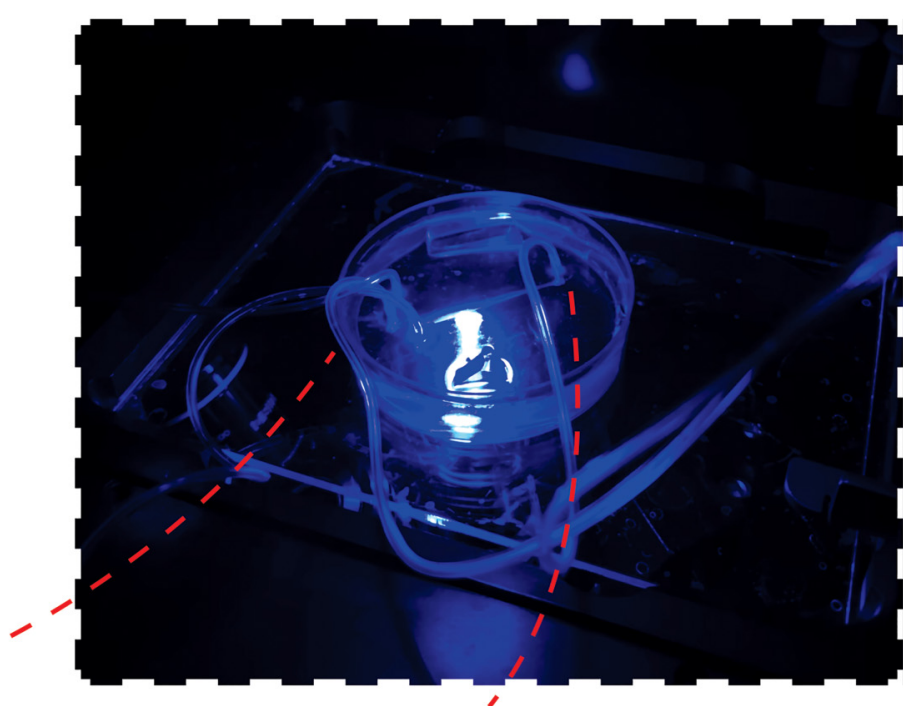

C)

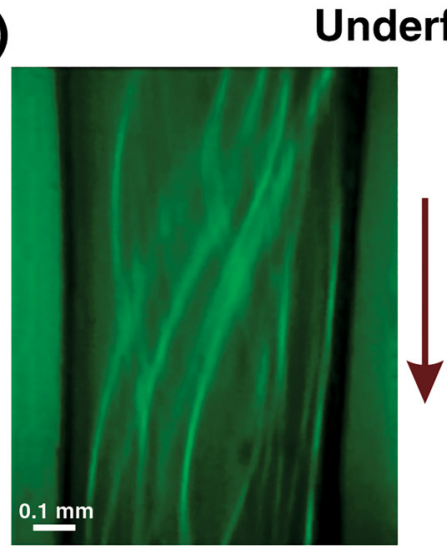

$10 \mu \mathrm{m}$
$\mathrm{Z}=2 \mathrm{~mm}$

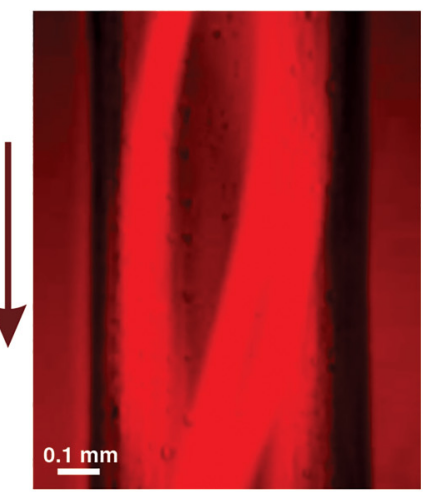

$100 \mu \mathrm{m}$

FIG. 5. (a) Experimental setup for fluorescent microscopy of micro-hydrocyclone. (b) Streamlines of the overflow for two different particle sizes of 10 and $100 \mu \mathrm{m}$. It is clear that only $10 \mu \mathrm{m}$ particles pass through the overflow. The images were taken at $Z=2 \mathrm{~mm}$ and $30 \mathrm{~mm}$ for underflow and overflow, respectively, following the same reference as used for simulation in Fig. 2. (c) Underflow streamlines for two different particle sizes of 10 and $100 \mu \mathrm{m}$. Almost all $100 \mu \mathrm{m}$ particles (efficiency >95\%) went through the underflow. 

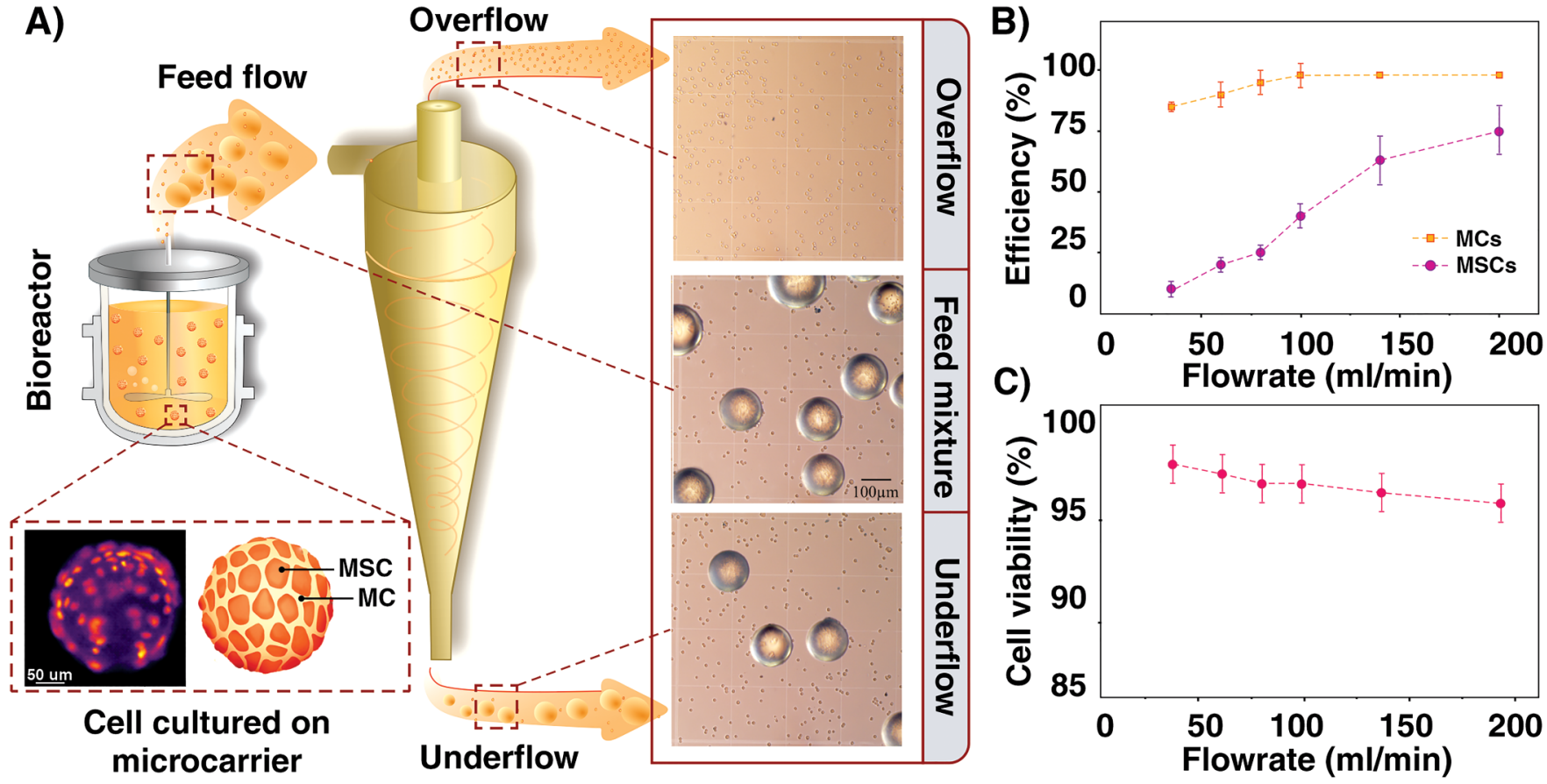

FIG. 6. (a) Schematic model of experimental setup which depicts the hemocytometer images of the feed mixture of MSCs and MCs, overflow sample showing MSCs only, and the underflow sample showing MSCs and concentrated MCs at $70 \mathrm{ml} / \mathrm{min}$. The inset also shows the MSCs cultured on MCs via experimental and schematic illustrations. (b) Comparison of efficiency curves for MCs and MSCs over a range of feed flow rates. By increasing the flow rate from 30 to $150 \mathrm{ml} / \mathrm{min}$, the separation efficiency of MSCs and MCs was enhanced, while the separation efficiency for MCs was up to $95 \%$. (c) Variation on cell viability for different flow rates. Cell viability for all flow rates was more than $95 \%$.

To take this a step further, as a proof of concept, we have used the "transparent" micro-hydrocyclone to investigate the trend of separation efficiency variation over a range of flow rates for the MCs and MSCs [Fig. 6(a)]. For the flow rates between 30 and $150 \mathrm{ml} / \mathrm{min}$, the separation efficiency of MSCs from MCs was increasingly enhanced, as was visually confirmed in Fig. 3. Therefore, at the flow rate $\mathrm{Q}=70 \mathrm{ml} / \mathrm{min}$, the cells exited through both outlets following the fluid flow, whereas up to $95 \%$ of the MCs were forced to exit through the underflow only. The viability of the cells was also monitored for each flow rate and no dramatic drop in viability was observed [Fig. 6(b)]. This shows that the high stresses inside the micro-hydrocyclone negligibly changed cell viability. At lower flow rates, efficiency of the cells slightly decreased, whereas there was a considerable enhancement at higher flow rates resulting in a higher cell concentration in the underflow. Therefore, for the fractionation or for the removal of the microcarriers from the mixture, the flow rate of $70 \mathrm{ml} / \mathrm{min}$ would be optimal. The cells were collected at the overflow and the cells lost to the underflow were recaptured by the recirculation of the underflow [Fig. 6(a)]. Cell recovery rate depends on the flow ratio, which is the fraction of the flow toward the underflow. For this design, the flow ratio was $60 \%$ which means $40 \%$ recovery of the cells to the overflow, for one passage. However, with the recirculation of the underflow stream, higher recovery rates $\sim 80 \%$ were achieved.
It can be concluded that the designed PDMS-based transparent micro-hydrocyclone can be used as a fast, continuous, reliable, and cheap alternative for various biotechnological applications. Furthermore, a transparent PDMS-based micro-hydrocyclone also allows real-time monitoring of the separation process and efficiency, which could be useful in industries as compared to repeated sample taking and counting. Surface transparency can also help to detect clogging or particle built up that could arise over time when dealing with such large-sized particles. Also, due to the simple geometry of the micro-hydrocyclone, the maintenance and cleaning of the device can be easy to carry out and monitor.

\section{CONCLUSION}

The main aim of this study was to propose a workflow for fabrication and flow visualization of a transparent PDMS-made microhydrocyclone. This is useful for next generation $3 \mathrm{D}$ devices that take advantage of additive manufacturing. In our approach, a new micro-hydrocyclone design was fabricated using a 3D-printed dissolvable mold and soft lithography with PDMS. This resulted in a transparent design wherein we were able to observe particle trajectories for different fluorescent and non-fluorescent particles could easily be identified at the entrance, middle, and outlets of the PDMS-made micro-hydrocyclone, making the efficiency 
determination and particle trajectory much easier. The experimental results also validated the simulation results and has a potential to reveal things that could not be observed with the simulation model due to the various assumptions and simplification. For instance, vortex fluctuations and bounce-like movement of the particles could not be observed in our numerical model since these simulations are performed at the steady-state conditions to save time and computational cost. The difference between experimental and numerical results (Fig. S2C in the supplementary material) is highlighting this matter too.

Furthermore, the high-speed imaging of bigger particles $(\sim 180 \mu \mathrm{m})$ also depicted the particles swirling inside the main micro-hydrocyclone body, enabling calculations of the rotation, angular velocity, and velocity magnitudes for the future investigations. The presence of turbulent eddies makes the theoretical and numerical analysis of the fluid/particle behavior complex, whereas the proposed method of direct observation takes into account all the real phenomena (including fabrication tolerances and surface roughness) within the $3 \mathrm{D}$ device. To showcase the potential application of this device, tests were conducted with living cells (e.g., separating them from polystyrene microcarriers). This produced encouraging results with more than $95 \%$ removal of the microparticles from the cell culture at the optimized flow rate of $\mathrm{Q}=70 \mathrm{ml} / \mathrm{min}$ and little loss of cell viability.

Based upon this study, it can be concluded that the ability of $3 \mathrm{D}$-printing to fabricate complex geometries can open up a lot of new avenues of research and development, such as microhydrocyclones. In such applications, using transparent materials, like PDMS, for these devices will enable biologists to dynamically monitor the operation of the device. These characteristics are crucial when it comes to commercialization of the microfluidic devices and for dealing with large volumes of cell cultures to process.

\section{SUPPLEMENTARY MATERIAL}

See the supplementary material for (1) PDMS box for the casting of the PDMS on the wax model, (2) (A) Simulated trajectories of 10 and $100 \mu \mathrm{m}$ particles through micro hydrocyclone at $\mathrm{v}=5 \mathrm{~m} / \mathrm{s}$, (B) the comparison of separation efficiency for the two experimental and numerical models; (3) cell proliferation analysis after passing through the device to confirm the cell viability; (4) high-speed camera movie for the particle trajectory at flow rates of 30 and $70 \mathrm{ml} / \mathrm{min}$; (5) fluorescent microscopy video of particles at flow rates of 10 and $70 \mathrm{ml} / \mathrm{min}$; and (6) fluorescent microscopy video of $10 \mu \mathrm{m}$ and $100 \mu \mathrm{m}$ particles at the underflow and overflow outlets.

\section{ACKNOWLEDGMENTS}

M.S.S. would like to acknowledge support of the Australian Government Research Training Program Scholarship for conducting this research at UNSW. M.E.W. would like to acknowledge support of the Australian Research Council through Discovery Project Grant Nos. DP170103704 and DP180103003 and the National Health and Medical Research Council through the Career Development Fellowship (No. APP1143377).

\section{DATA AVAILABILITY}

The data that support the findings of this study are available from the corresponding author upon reasonable request.

\section{REFERENCES}

'Z. Luo, M. Fan, Y. Zhao, X. Tao, Q. Chen, and Z. Chen, Powder Technol. 187, 119-123 (2008).

${ }^{2}$ X. Qu, P. J. J. Alvarez, and Q. Li, Water Res. 47, 3931-3946 (2013).

${ }^{3}$ M. E. Warkiani, C.-P. Lou, H.-B. Liu, and H.-Q. Gong, Biomed. Microdevices 14, 669-677 (2012).

${ }^{4}$ M. Ebrahimi Warkiani, C.-P. Lou, and H.-Q. Gong, Biomicrofluidics 5, 036504 (2011).

${ }^{\mathbf{5}}$ H. Mohamed, M. Murray, J. N. Turner, and M. Caggana, J. Chromatogr. A 1216, 8289-8295 (2009).

${ }^{6}$ S. Razavi Bazaz, A. Mashhadian, A. Ehsani, S. Saha, T. Krueger and M. Ebrahimi Warkiani, Lab Chip 20, 1023 (2020).

${ }^{7}$ L. Chen, M. E. Warkiani, H.-B. Liu, and H.-Q. Gong, J. Micromech. Microeng. 20, 075005 (2010).

${ }^{8}$ J. Zhang, S. Yan, D. Yuan, G. Alici, N.-T. Nguyen, M. Ebrahimi Warkiani, and W. Li, Lab Chip 16, 10-34 (2016).

${ }^{9}$ P. Sajeesh and A. K. Sen, Microfluid. Nanofluid. 17, 1-52 (2014).

${ }^{10}$ S. Razavi Bazaz, A. Mashhadian, A. Ehsani, S. C. Saha, T. Krüger, and M. Ebrahimi Warkiani, Lab Chip 20, 1023-1048 (2020).

${ }^{11}$ M. Kersaudy-Kerhoas, R. Dhariwal, M. P. Desmulliez, and L. Jouvet, Microfluid. Nanofluid. 8, 105-114 (2010).

${ }^{12}$ M. Yamada and M. Seki, Lab Chip 5, 1233-1239 (2005).

${ }^{13}$ M. Yamada and M. Seki, Anal. Chem. 78, 1357-1362 (2006).

${ }^{14}$ L. R. Huang, E. C. Cox, R. H. Austin, and J. C. Sturm, Science 304, 987-990 (2004).

${ }^{15}$ J. Takagi, M. Yamada, M. Yasuda, and M. Seki, Lab Chip 5, 778-784 (2005).

${ }^{16}$ M. Yamada, M. Nakashima, and M. Seki, Anal. Chem. 76, 5465-5471 (2004).

${ }^{17}$ D. Di Carlo, Lab Chip 9, 3038-3046 (2009).

${ }^{18}$ M. E. Warkiani, A. K. P. Tay, G. Guan, and J. Han, Sci. Rep. 5, 11018 (2015).

${ }^{19}$ A. S. Rzhevskiy, S. Razavi Bazaz, L. Ding, A. Kapitannikova, N. Sayyadi, D. Campbell, B. Walsh, D. Gillatt, M. Ebrahimi Warkiani, and A. V. Zvyagin, Cancers 12, 81 (2019).

${ }^{20}$ A. Mashhadian and A. Shamloo, Anal. Chim. Acta 1083, 137-149 (2019).

${ }^{21}$ J. Zhou and I. Papautsky, Lab Chip 19, 3416-3426 (2019).

${ }^{22}$ J. Zhou, C. Tu, Y. Liang, B. Huang, Y. Fang, X. Liang, I. Papautsky, and X. Ye, Sci. Rep. 8, 9411 (2018).

${ }^{23}$ R. Martinez-Duarte, R. A. Gorkin III, K. Abi-Samra, and M. J. Madou, Lab Chip 10, 1030-1043 (2010).

${ }^{24}$ J. Zhang, Q. Guo, M. Liu, and J. Yang, J. Micromech. Microeng. 18, 125025 (2008).

${ }^{\mathbf{2 5}}$ S. Waheed, J. M. Cabot, N. P. Macdonald, T. Lewis, R. M. Guijt, B. Paull, and M. C. Breadmore, Lab Chip 16, 1993-2013 (2016).

${ }^{\mathbf{2 6}} \mathrm{J}$. Yu and Y. Kim, Desalin. Water Treat. 57, 629-635 (2016).

${ }^{27}$ Y. X. Xu, Y. Liu, Y. H. Zhang, X. J. Yang, and H. L. Wang, Chem. Eng. Technol. 39, 567-575 (2016).

${ }^{28}$ Y. Huang, H. L. Wang, Y. Q. Chen, Y. H. Zhang, Q. Yang, Z. S. Bai, and L. Ma, Sci. Rep. 7, 2678 (2017).

${ }^{29}$ P. Bhardwaj, P. Bagdi, and A. Sen, Lab Chip 11, 4012-4021 (2011).

${ }^{30}$ I. C. Bicalho, J. L. Mognon, J. Shimoyama, C. H. Ataíde, and C. R. Duarte, Sep. Sci. Technol. 48, 915-922 (2013).

${ }^{31}$ M. Shakeel Syed, M. Rafeie, R. Henderson, D. Vandamme, M. Asadnia, and M. Ebrahimi Warkiani, Lab Chip 17, 2459-2469 (2017).

${ }^{32}$ D. Vega-Garcia, P. R. Brito-Parada, and J. J. Cilliers, Chem. Eng. J. 350, 653-659 (2018).

${ }^{33}$ I. W. Bettinardi, A. Castan, R. A. Medronho, and L. R. Castilho, Biotechnol. Bioeng. 117, 1915-1928 (2020). 
${ }^{34}$ T. Lawson, D. E. Kehoe, A. C. Schnitzler, P. J. Rapiejko, K. A. Der, K. Philbrick, S. Punreddy, S. Rigby, R. Smith, Q. Feng, J. R. Murrell, and M. S. Rook, Biochem. Eng. J. 120, 49-62 (2017).

${ }^{35}$ A. Mizukami and K. Swiech, Stem Cells Int. 2018, 13 (2018).

${ }^{36}$ B. J. Kim, T. Zhao, L. Young, P. Zhou, and M. L. Shuler, Biotechnol. Bioeng. 109, 137-145 (2012).

${ }^{37}$ A. W. Nienow, Q. A. Rafiq, K. Coopman, and C. J. Hewitt, Biochem. Eng. J. 85, 79-88 (2014).

${ }^{38}$ S. Hassan, A. S. Simaria, H. Varadaraju, S. Gupta, K. Warren, and S. S. Farid, Regen. Med. 10, 591-609 (2015).

${ }^{39}$ A. Naderi, N. Bhattacharjee, and A. Folch, Annu. Rev. Biomed. Eng. 21, 325-364 (2019).

${ }^{40}$ S. Razavi Bazaz, N. Kashaninejad, S. Azadi, K. Patel, M. Asadnia, D. Jin, and M. Ebrahimi Warkiani, Adv. Mater. Technol. 4, 1900425 (2019).

${ }^{41}$ N. Bhattacharjee, C. Parra-Cabrera, Y. T. Kim, A. P. Kuo, and A. Folch, Adv. Mater. 30, 1800001 (2018).

${ }^{42}$ Y.-S. Lee, N. Bhattacharjee, and A. Folch, Lab Chip 18, 1207-1214 (2018).

${ }^{43}$ M. S. Syed et al., Sep. Purif. Technol. 254, 117610, (2021).

${ }^{44}$ M. A. Raoufi, S. R. Bazaz, H. Niazmand, O. Rouhi, M. Asadnia, A. Razmjou, and M. E. Warkiani, Soft Matter 16(10), 2448-2459 (2020).

${ }^{45} \mathrm{~V}$. Saggiomo and A. H. Velders, Adv. Sci. 2, 1500125 (2015).

${ }^{46} \mathrm{~J}$. Yang, K. Li, L. Zhu, and W. Tang, RSC Adv. 7, 3313-3320 (2017).

${ }^{47}$ A. K. Au, N. Bhattacharjee, L. F. Horowitz, T. C. Chang, and A. Folch, Lab Chip 15, 1934-1941 (2015).

${ }^{48}$ Z.-S. Bai, H.-L. Wang, and S.-T. Tu, Miner. Eng. 22, 319-323 (2009).

${ }^{49}$ Y. Fan, J. Wang, Z. Bai, J. Wang, and H. Wang, Sep. Purif. Technol. 149, 156-164 (2015).

${ }^{50}$ J. Wang, Z. Bai, Q. Yang, Y. Fan, and H. Wang, Sep. Purif. Technol. 163, 120-127 (2016).
${ }^{51}$ Z. Stęgowski and E. Nowak, Nukleonika 52(3), 115-123 (2007), see http://yadda.icm.edu.pl/baztech/element/bwmeta1.element.baztech-article-BUJ60023-0006.

${ }^{52}$ C. Song, B. Pei, M. Jiang, B. Wang, D. Xu, and Y. Chen, Powder Technol. 294, 437-448 (2016).

${ }^{53}$ R. H. Perry, in Chemical Engineering Handbook, edited by R. H. Perry and D. Green (McGraw-Hill, New York, 1984).

${ }^{54}$ K. Rietema and S. I. R. Maatschappij, Chem. Eng. Sci. 15, 298-302 (1961).

${ }^{55}$ W. O. Witbeck and D. R. Woods, Can. J. Chem. Eng. 62, 91-98 (1984).

${ }^{56}$ S. Schütz, G. Gorbach, and M. Piesche, Chem. Eng. Sci. 64, 3935-3952 (2009).

${ }^{57}$ P. Bagdi, P. Bhardwaj, and A. Sen, J. Fluids Eng. 134, 021105 (2012).

${ }^{58}$ R. Medronho, J. Schuetze, and W. Deckwer, Lat. Am. Appl. Res. 35, 1-8 (2005).

${ }^{59}$ D. Vega-Garcia, J. J. Cilliers, and P. R. Brito-Parada, Sep. Purif. Technol. 251, 117253 (2020).

${ }^{60}$ H. H. Al-Kayiem, H. Osei, F. M. Hashim, and J. E. Hamza, J. Pet. Explor. Prod. Technol. 9(4), 2943-2952 (2019).

${ }^{61}$ H. Osei, H. Al-Kayiem and O. Ahmed 11, 12159-12164 (2016), see https:// scholar.google.com/scholar?hl=en\&as_sdt $=0 \% 2 \mathrm{C} 5 \& \mathrm{q}=\mathrm{O}$ sei $\% 2 \mathrm{C}+\mathrm{H} . \% 2 \mathrm{C}+\mathrm{Al}-$ Kayiem\%2C+H.H.\%2C+Osman\%2C+A.B.+Flow+dynamics+behaviour+of +a + novel+liquid-liquid+hydrocyclone+withvarying+upper+cylindrical+lengths + and +number+of+inlets+. + ARPN + Journal + of + Engineering + and + Applied + Sciences $+11 \% 2820 \% 29 \% 2 \mathrm{C}+2016 .+$ pp. $+12159-12164 \&$ btn G $=$.

${ }^{62}$ L. Abdollahzadeh, M. Habibian, R. Etezazian, and S. Naseri, Powder Technol. 283, 294-301 (2015).

${ }^{63}$ C.-Y. Hsu, S.-J. Wu, and R.-M. Wu, Tamkang J. Sci. Eng. 14, 65-70 (2011).

${ }^{64}$ Z.-B. Wang, L.-Y. Chu, W.-M. Chen, and S.-G. Wang, Chem. Eng. J. 138, 1-9 (2008).

${ }^{65}$ R. C. Pinto, R. A. Medronho, and L. R. Castilho, Cytotechnology 56, 57-67 (2008).

${ }^{66} \mathrm{H}$. Tavassoli, S. N. Alhosseini, A. Tay, P. P. Chan, S. K. W. Oh, and M. E. Warkiani, Biomaterials 181, 333 (2018). 